\title{
Medical Image of the Week: Bronchopleural Fistula
}

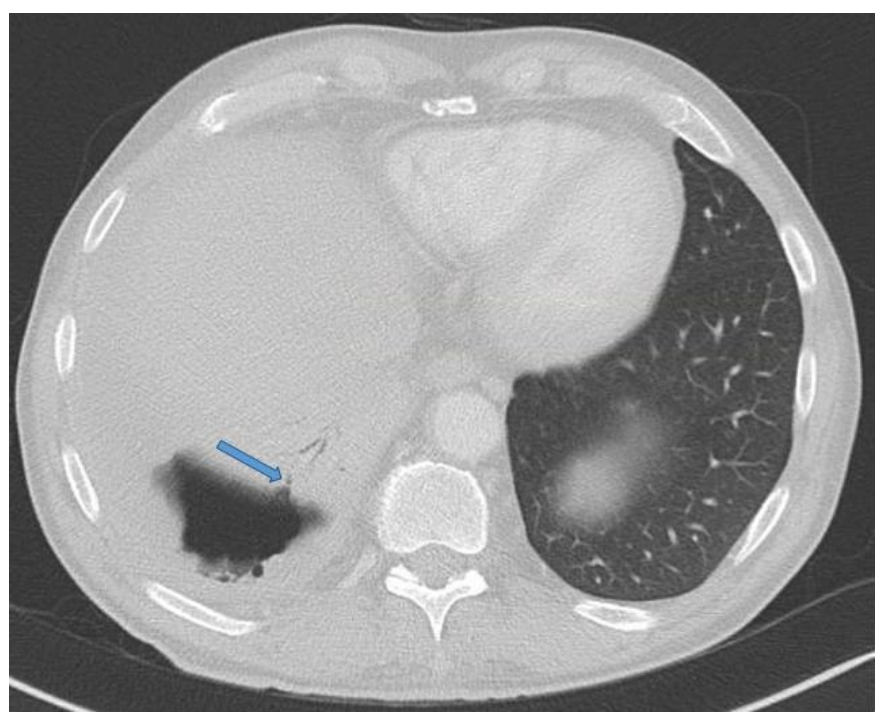

Figure 1. The blue arrow indicates a fistulous communication between bronchus and the pleura.

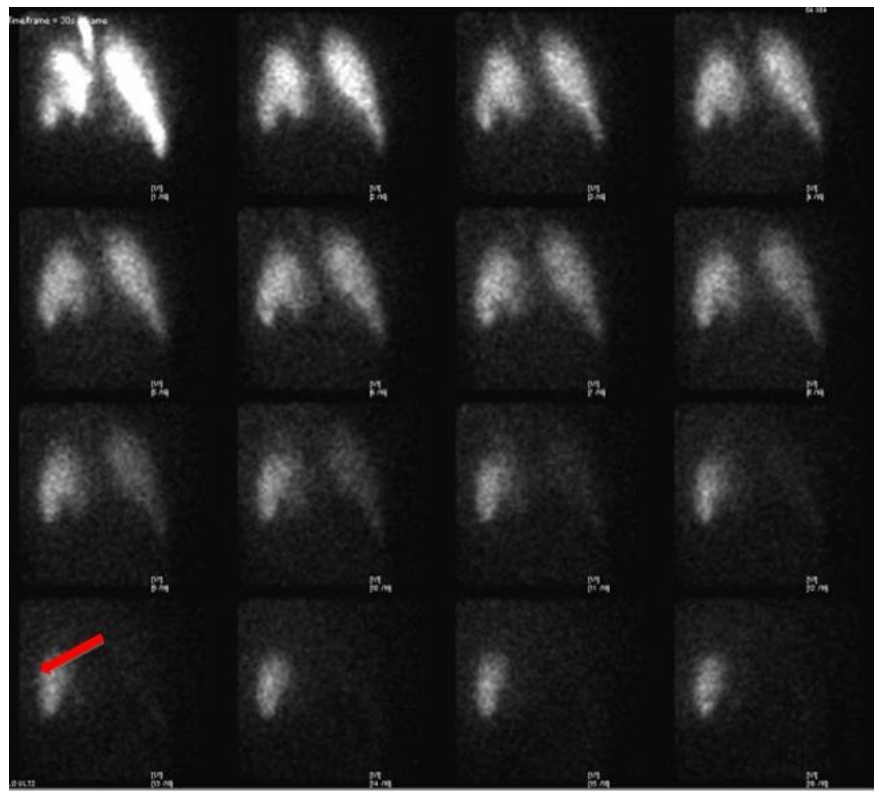

Figure 2. Red arrow indicates radiotracer trapping in the pleural space and adjacent right anterior lower lung field.

A 58-year-old man with past medical history significant for chronic smoking and seizures was referred to the emergency room after a chest $x$-ray done by his primary care physician for evaluation of cough showed a hydropneumothorax. His symptoms included dry cough for past 2 months without fever, chills or other associated symptoms. He did not have any thoracic procedures performed and had no past history 
of recurrent infections. He was hemodynamically stable. Physical examination was only significant with decreased breath sounds on the right side of the chest.

Thoracic CT with contrast was performed which showed complete collapse of the right lower lobe, near complete collapse of right middle lobe as well as an air-fluid level. There was a suspicion of a direct communication between bronchi and pleural space at the posterior lateral margin of the collapsed right lower lobe (Figure 1). The presence of bronchopleural fistula (BPF) was confirmed with the help of lung scintigraphy, which showed trapping of radiotracer in the right lung at a location consistent with the chest CT findings of BPF (Figure 2). The patient was admitted and extensive infectious work up was negative. Biopsy of the right pleura was negative for malignancy. He underwent video-assisted thoracoscopic surgery (VATS) decortication of his right lung and was stable on subsequent follow up with complete resolution of his cough.

BPF has mortality rate between 18 to $67 \%$ (1). CT of the chest can occasionally demonstrate a direct fistulous communication, as seen in our case. However, Westcott and Volpe (2) have shown sensitivity of CT chest to be $50 \%$. Other useful noninvasive imaging techniques include lung scintigraphy. In this modality, the fistula is identified by seeing a trapping of radioisotope labeled gas as there is no mechanism for a clearance of gas after its diffusion into the pleural space. Raja et al. (3) demonstrated that lung scintigraphy had $83 \%$ sensitivity and $100 \%$ specificity in diagnosing BPF.

As seen in this case lung nuclear scintigraphy offers a cheaper, more sensitive, and less invasive approach to diagnosis of BPF.

Hem Desai MD, MPH and Anthony Witten DO

Department of Internal Medicine

University of Arizona

Tucson, AZ USA

\section{References}

1. Hollaus PH, Lax F, el-Nashef BB, Hauck HH, Lucciarini P, Pridun NS. Natural history of bronchopleural fistula after pneumonectomy: a review of 96 cases. Ann Thorac Surg. 1997 May;63(5):1391-6; discussion 1396-7. [CrossRef] [PubMed]

2. Westcott JL, Volpe JP. Peripheral bronchopleural fistula: CT evaluation in 20 patients with pneumonia, empyema, or postoperative air leak. Radiology. 1995 Jul;196(1):175-81. [CrossRef] [PubMed]

3. Raja S, Rice TW, Neumann DR, Saha GB, Khandekar S, Maclntyre WJ, Go RT. Scintigraphic detection of post-pneumonectomy bronchopleural fistulae. Eur J Nucl Med. 1999 Mar;26(3):215-9. [CrossRef] [PubMed] 